\title{
Alternate Environmentally Friendly De-Painting Process for Aircraft Structures-Atmospheric Plasma
}

\author{
Ali Merati, Marko Yanishevsky, Tessa Despinic, Philip Lo, Vladimir Pankov \\ National Research Council Canada, NRC Aerospace, Ottawa, Canada \\ Email: ali.merati@nrc-cnrc.gc.ca
}

How to cite this paper: Merati, A., Yanishevsky, M., Despinic, T., Lo, P. and Pankov, V. (2017) Alternate Environmentally Friendly De-Painting Process for Aircraft Structures-Atmospheric Plasma. Journal of Minerals and Materials Characterization and Engineering, 5, 223-235.

https://doi.org/10.4236/jmmce.2017.54019

Received: June 16, 2017

Accepted: July 4, 2017

Published: July 7, 2017

Copyright @ 2017 by authors and Scientific Research Publishing Inc. This work is licensed under the Creative Commons Attribution International License (CC BY 4.0).

http://creativecommons.org/licenses/by/4.0/

(c) (†) Open Access

\begin{abstract}
During the lifetime of aircraft structures, paint removal and re-application cycles are conducted to restore appearance and to enable inspection for fatigue cracks and corrosion damages. Current paint removals processes, including chemical and abrasive media blasting, yield large amounts of volatile organic compounds and hazardous air pollutants, and generate large quantities of waste which require proper disposal/treatment. They also have the potential to mask surface cracks and decrease the effectiveness of Liquid Penetrant Inspections (LPI). This study compares current paint stripping methods to a novel Atmospheric Plasma (AP) system, which claims to be a more environmentally friendly method. Aluminium and steel coupons were prepared with military aircraft quality topcoat and primer paint schemes and subjected to the three aforementioned paint stripping processes. The results were then characterized in terms of paint removal effectiveness, potential damages to the metallic substrates, and effect on the LPI process. Hardness and conductivity measurements as well as metallographic sectioning and microscopy, were used to characterize the stripped samples. The results indicated that AP had no negative ramifications on detecting fatigue cracks in the substrates, and did not alter the temper, mechanical properties of the aerospace alloys studied. The AP process has the potential to replace current hazardous and less environmentally friendly paint removal methods; though a full systematic qualification and evaluation process is still required for it to be considered as an accepted industrial paint removal process.
\end{abstract}

\section{Keywords}

Atmospheric Plasma, Paint Stripping, Chemical Stripping, Media Blast, Non-Destructive Testing (NDT), Liquid Penetrant Inspection (LPI), Fatigue Cracks, Aerospace 


\section{Introduction}

The conventional methods for removing paint employed throughout the Canadian Forces include environmentally unfriendly chemical stripping and abrasive media blasting [1] [2]. Chemical stripping is lengthy (typically 24 hours) and involves the use of hazardous chemicals, which are typically high in Volatile Organic Compounds (VOC) and Hazardous Air Pollutants (HAP). Long term exposure to these chemicals, which are considered carcinogens and toxins, also pose risks to worker health [3].

Abrasive media blasting typically results in a substantial quantity of solid waste consisting of paint and blast residues. Both types of wastes from chemical and media blasting are subject to control under increasingly stringent environmental and safety regulations and their disposal is costly. The LPI process is well suited for revealing discontinuities that are open to the surface of solid materials (essentially nonporous materials) and is extensively used for the inspection of products made of different materials: metals, ceramics, and plastics.

Paint removal methods can adversely affect the effectiveness of routine surface inspections such as LPI [4], if entrapped media or residues mask surface cracks. Entrapped residues may plug cracks and prevent infiltration of penetrant solution [5].

The new Atmospheric Plasma (AP) paint removal process claims to be environmentally benign utilizing only electricity and compressed air as the feed gas for operation. The process is done in a working chamber that requires an exhaust and a filtration system. The power supply in the AP process produces a high frequency electromagnetic field to generate "cold" plasma [3]. The plasma generates a high velocity flow of chemically active nitrogen and oxygen radicals. The chemical energy oxidizes the organic component found in paints and coatings, converting much of the removed paint into harmless gases such as water vapour, $\mathrm{CO}_{2}$, and solid residue.

The objective in this study was to compare the effectiveness of the AP process with that of chemical and Type VII media blasting methods in removing the paint on aluminium panels with cracks, and their effect on crack detectability. LPI was employed after the paint removal processes and the cracks were measured before and after for comparative analysis.

\section{Materials and Procedure}

The materials used in the investigation were 3003-H14 aluminium and SAE 1008 steel, as well as large panels of heat-treatable aerospace aluminium alloys 2024T3 and 7075-T6. They were prepared with coatings of paint and primer according to aerospace painting protocols [6] [7]. Each coupon was coated with yellow epoxy-polyamide (MILGUARD-23377) primer with a thickness range of 45 - 80 $\mu \mathrm{m}$ and a grey polyurethane coating (MIL-PRF-85285D) topcoat with a thickness range of $30-50 \mu \mathrm{m}$ [8] [9]. Figure 1(a) and Figure 1(b) show the cross section for the 2024-T3 aluminium panel in the regions of maximum and 


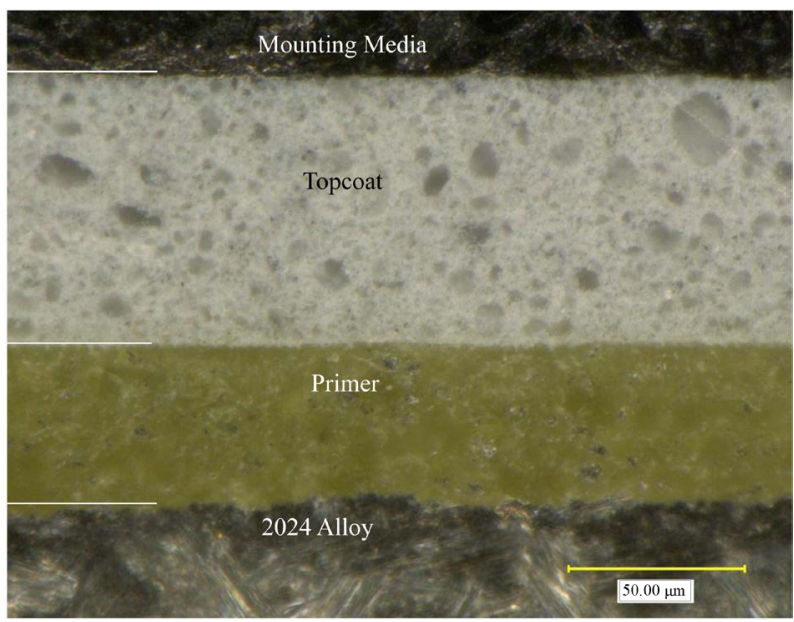

(a)

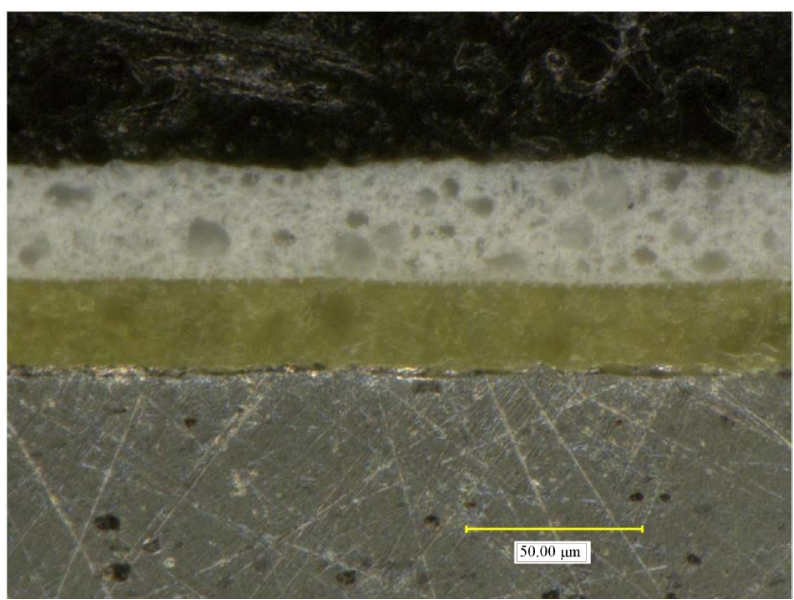

(b)

Figure 1. Cross sectional views of 2024-T3 aluminium panel: (a) cross section in the location of the maximum paint thickness; and (b) cross section in the location of minimum paint thickness (the order of legends follows the same as 1a).

minimum total paint thicknesses, which were about $115 \mu \mathrm{m}$ and $55 \mu \mathrm{m}$, respectively. For the large panels of 2024-T3 and 7075-T6, after coating, fatigue cracks were introduced to investigate the effects of paint removal process on crack detectability by LPI.

The panel sections with and without cracks were exposed to the three paint removal processes: Atmospheric Plasma, starch-acrylic media (Type VII) blasting, and chemical solution. Once each paint stripping process was completed, each panel section was visually inspected for the extent of paint removal and damages, such as burns or warping. Cracks were then examined in more detail for possible presence of residue entrapped in the cracks and if the residue affected the detectability of LPI. All panel sections were then inspected by LPI to assess and compare the three paint removal techniques.

\section{Results and Discussion}

The three paint removal processes were compared to determine if AP paint 
stripping is a viable alternative to current paint stripping methods used by the Canadian Forces. It should be noted that the conductivity and hardness of the aluminium coupons was measured before and after the paint removal treatments as a means of determining whether the coupons had been subjected to excessive heat during the paint removal process [10] [11]. Obviously, the change of temper was only checked for the two heat-treatable aluminium alloys of 2024-T3 and 7075-T6. The Al 3003-H14 is not a heat treatable alloy.

\subsection{Atmospheric Plasma}

The power supply in the Atmospheric Plasma process can produce a high frequency electromagnetic field to generate relatively cold plasma as well as hot plasmas operating at hundreds or thousands degrees above ambient [3]. Figure 2(a) and Figure 2(b) are indicative of very high temperatures (up to $400^{\circ} \mathrm{C}$ ) reached at front and the charred paper stickers on the back of the coupon. The process parameters should be suitably selected in order to produce a relatively low thermal energy exposure "cold process" that does not damage temperaturesensitive substructures, such as heat-treated aerospace aluminium alloys during the depainting process. Understandably, prior to comparing the novel AP

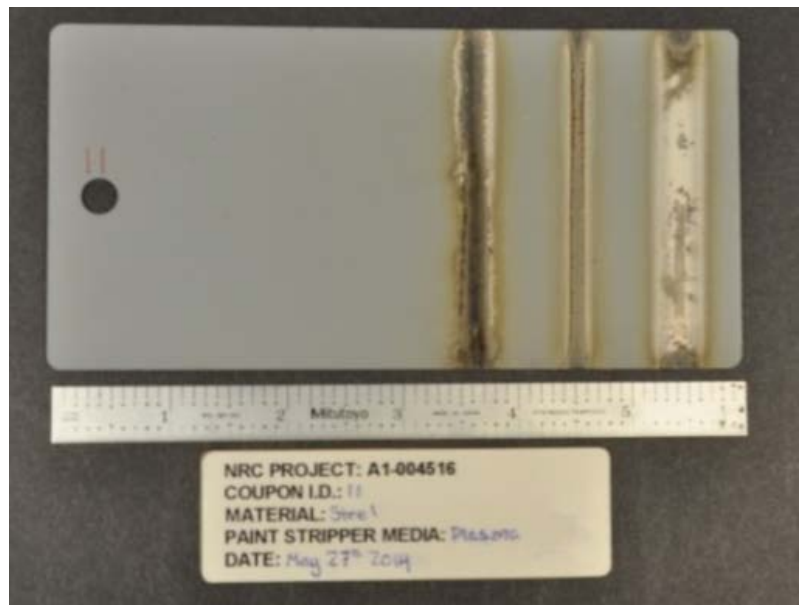

(a)

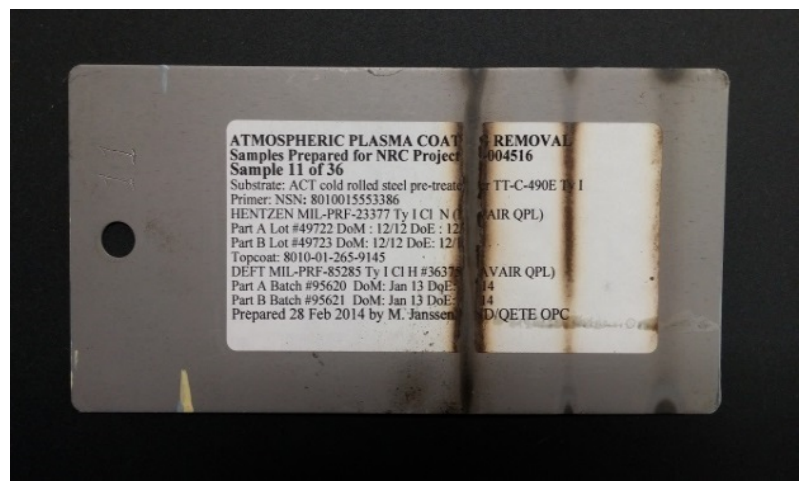

(b)

Figure 2. Post-strip view of the SAE 1008 steel coupon: (a) rear view indicating the charred paper sticker on the back of coupon. 
process to current conventional methods chemical and media blast, it was necessary to establish a working envelope of process parameters in order to remove the coating without damaging the substrate.

\subsubsection{Optimum Parameters}

The parameters that were examined were the height and speed of the plasma pen, as well as the number of individual stripes (passes) over the same area. The objective was to identify the boundary conditions for effective, ineffective, and damaging parameter envelop, which involved trial of the minimum and maximum parameters to establish a region of appropriate process parameters that would provide effective paint stripping without damaging or deforming the substrate material. The APS PlasmaFlux paint removal system was used to strip multiple panels of aluminium alloys with topcoat and primer under various process conditions through the analysis of possible damage to the substrate material and effectiveness of paint removal. Details of the operating procedure for APS PlasmaFlux system are documented in previous reports [1] [2] [12].

Through graphical analysis, as shown in Figure 3, the general relationships between the height, speed and number of passes of the plasma gun were developed. When the height of the plasma nozzle is kept constant, the speed of the nozzle affects the amount of paint removed by each pass of the gun. As the speed decreases, an incrementally larger thickness of paint is removed.

A similar behaviour was observed while maintaining the travel speed of the nozzle constant and altering the height of the plasma nozzle. A decrease in height of nozzle tip away from the specimen resulted in more paint being stripped, as shown in Figure 4.

It should be noted that regardless of the parameters used, the AP process

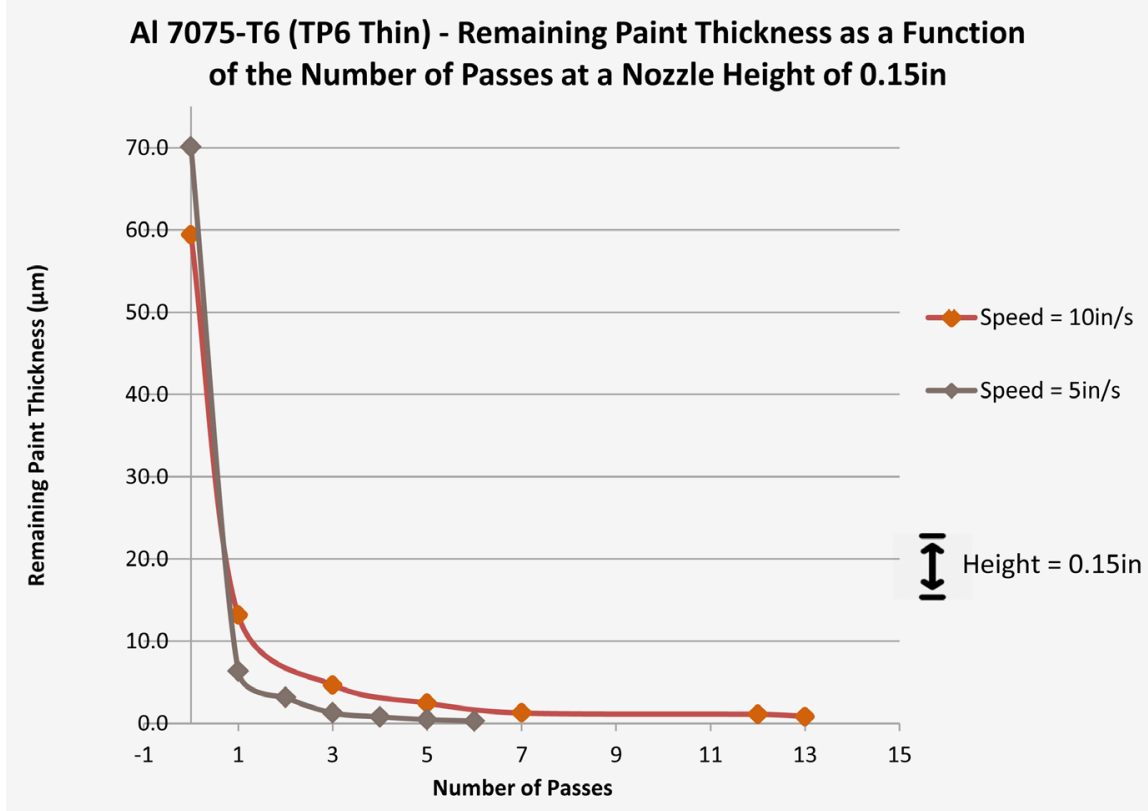

Figure 3. Graph of Al 7075-T6 (TP6 Thin, 0.0625" $1.5 \mathrm{~mm}$ thick) illustrating that slower speeds strips paint faster with the height held constant. 


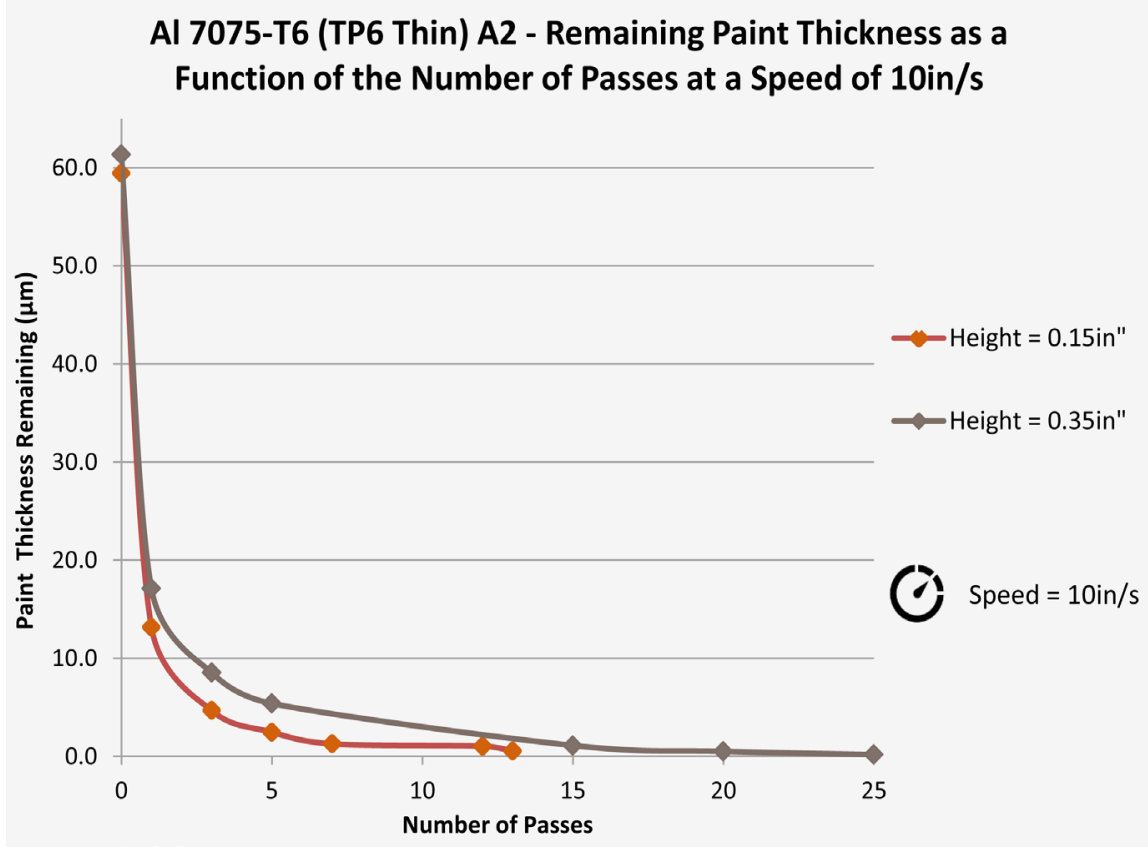

Figure 4. Graph of Al 7075-T6 Thin (0.0625" $1.5 \mathrm{~mm}$ thick); illustrating that using a lower height will strip paint faster when the speed is held constant.

strips more than half of the total paint in the first pass and often revealed the primer underneath the topcoat. Graphing the results yielded an exponentially decaying relationship between the thickness of paint remaining on the panel and the number of passes performed, conveying a plateau curve for the amount of paint stripped after the first few passes.

With all of the paint stripping trials performed and the data plotted, a working envelope of parameters was identified. Essentially plasma gun height values ranging from 0.2 " $(5.1 \mathrm{~mm})$ up to 1.2 " $(30.5 \mathrm{~mm})$ are practical and effective for paint stripping. Similar outcomes can be attained by picking the plasma gun translational speed and adjusting the gun height depending on how quickly the paint needs to be stripped. Figure 5 provides a graphical representation of all paint stripping tests that were performed. It displays the parameters which damage the surface, those that have no effect on the surface, and those that are effective in paint stripping. It is noteworthy that for the thick plates, the working envelope was extending to include the very low speeds and low heights of the nozzle, as can be seen in the lower left corner of graph in Figure 5. The larger volume of the aluminium substrate material associated with the thick plates likely acted as a heat sink, explaining why there was no measurable damage observed to the thick substrate plates.

Figure 6 is essentially a colour map of Figure 5, plotting the effective, damaging, and ineffective regions with the number of passes taken into account. The colour gradient from red to blue shows the intensity and amount of paint stripped from the greatest to the least, within the studied range of process parameters.

After establishing the optimal and safe working region, the AP de-painting 


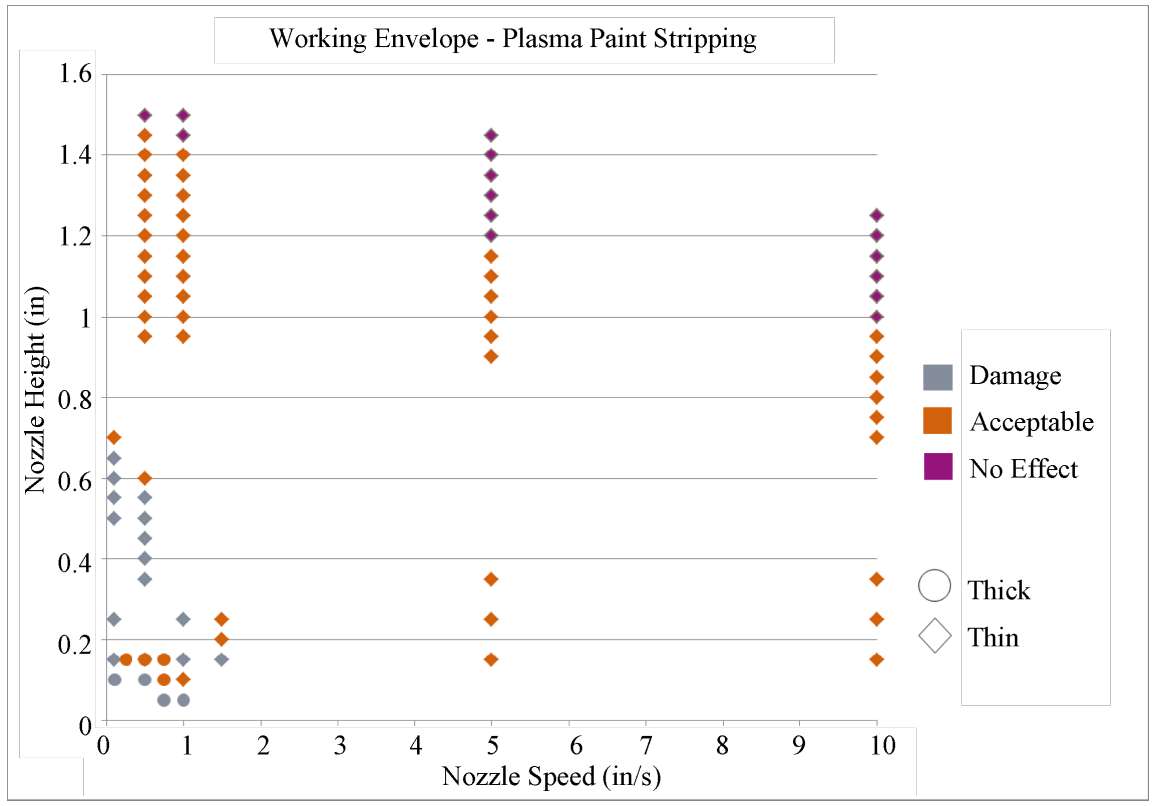

Figure 5. 2D graph of damaging, acceptable and ineffective limits using 3 passes of the plasma pen.

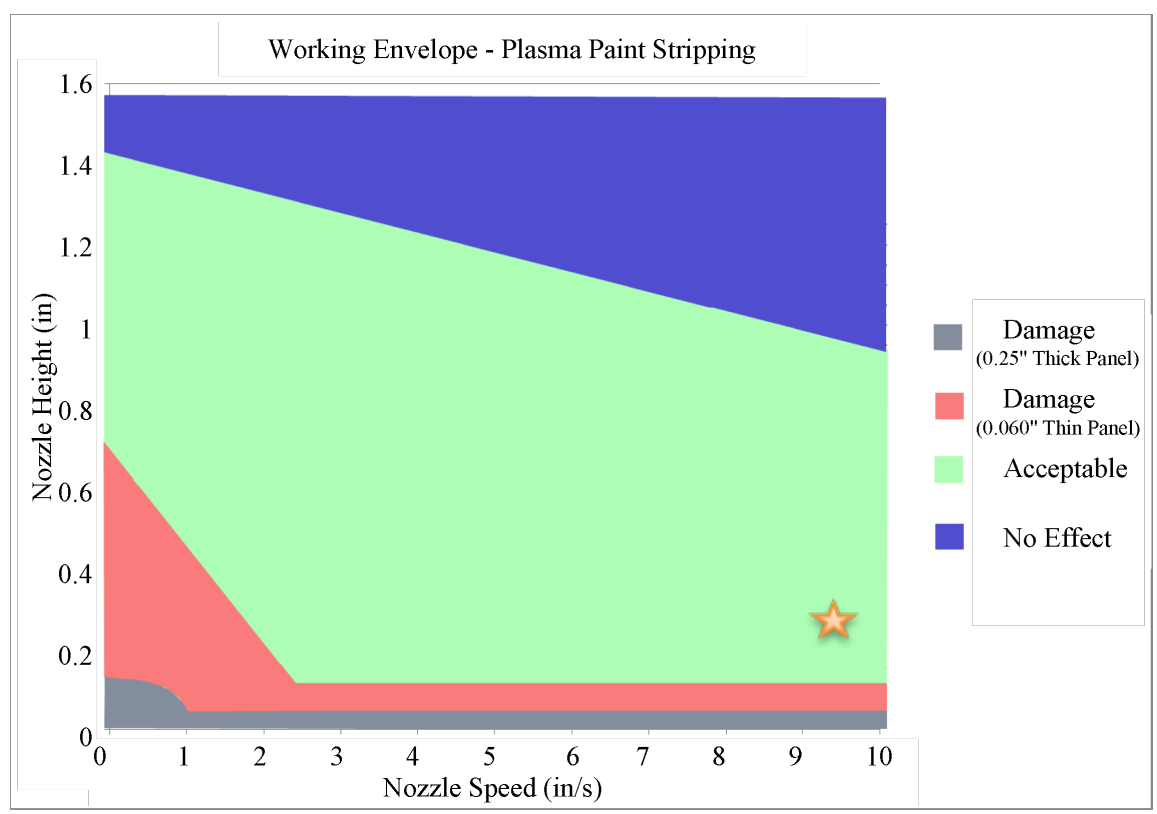

Figure 6. Map of effective (acceptable), damaging and ineffective parameters for the paint stripping machine on the specified paint scheme. The star indicates the selected parameters that were used in the comparative study.

process was compared with conventional methods of chemical solution and media blasting, using the process parameters identified in Table 1. The AP parameter combination selected in this study is specified by the star in Figure 6 .

\subsubsection{Atmospheric Plasma Performance}

As can be seen in Figure 7, the result of de-painted coupons (without cracks) showed no visually apparent damage caused by the plasma plume. Although in 


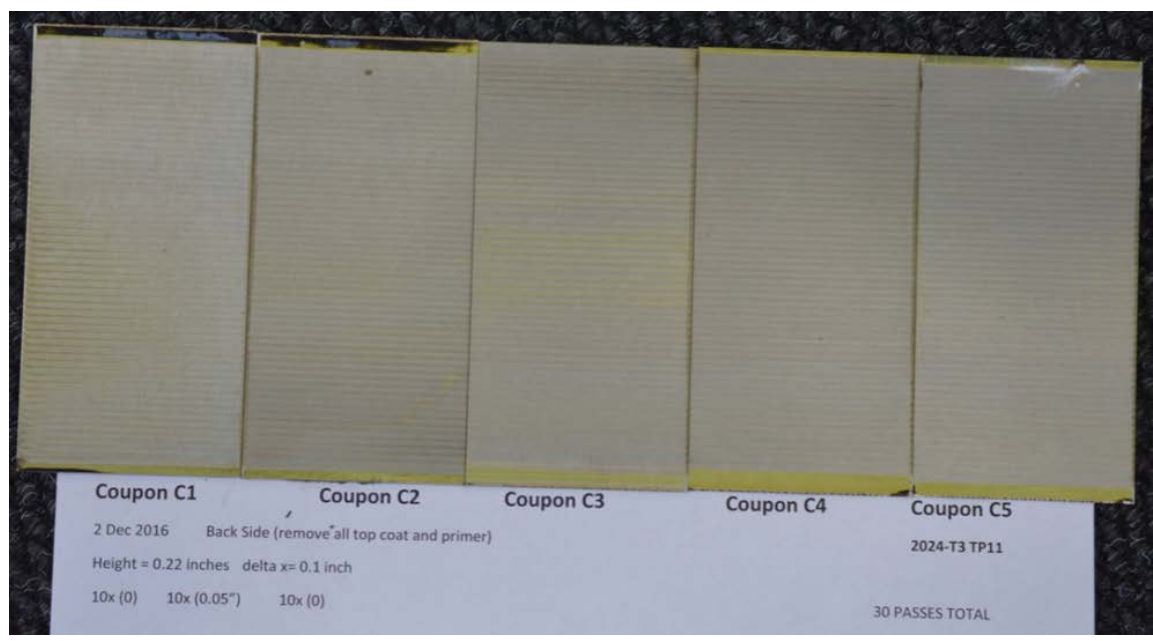

Figure 7. Evidence of the AP de-painting process on 5 coupons for almost complete removal of top coat and primer.

Table 1. Selected process parameters used in the de-painting comparative study.

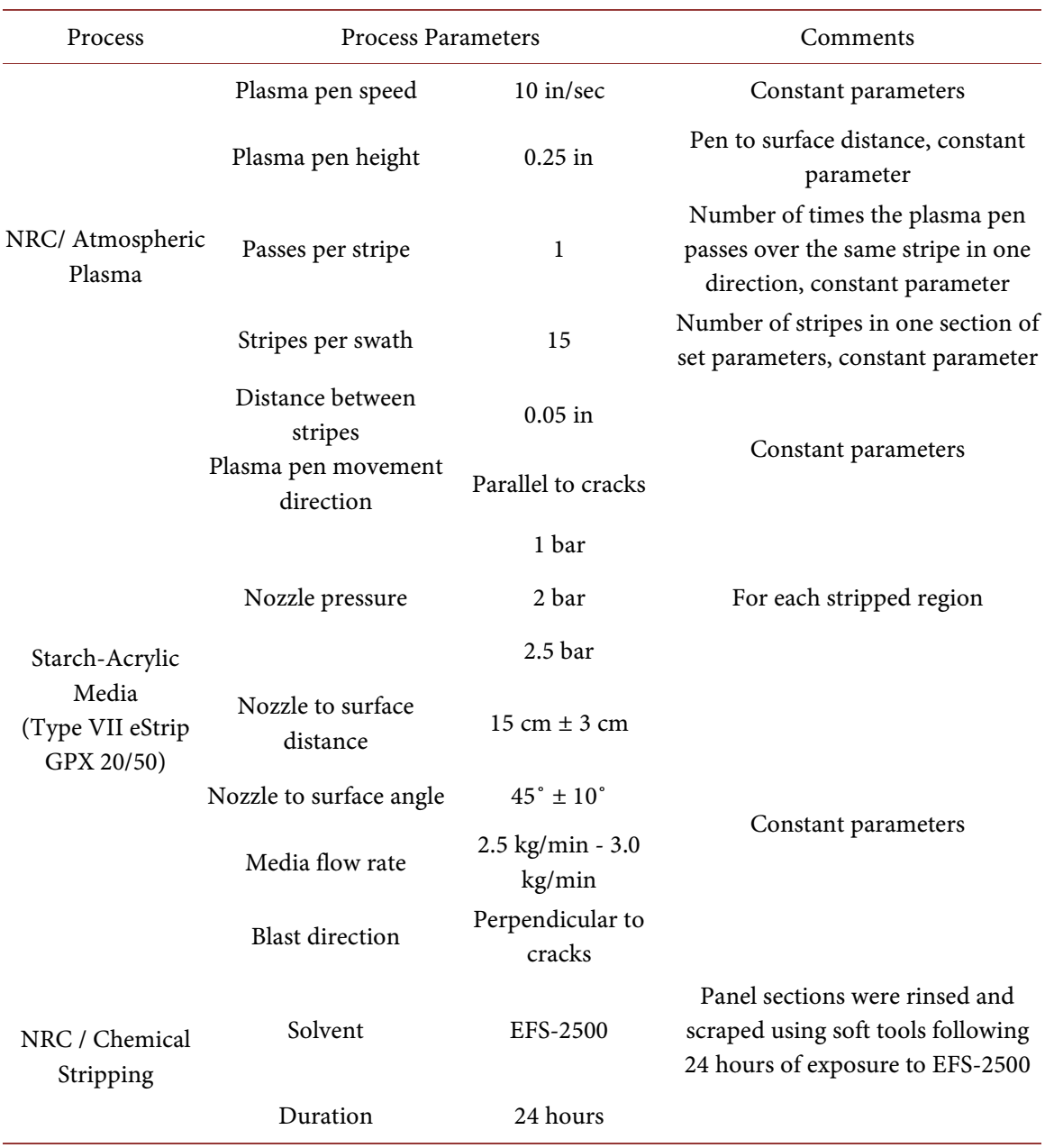

the case of AP process, the intent was not a total removal of primer; however, as can be seen in Figure 7, the process was capable of completely stripping the primer off. 
The results of the process for the coupons in presence of cracks were also encouraging. Figure 8 is an example of optical analysis suggesting a clean crack opening with no obvious debris entrapped inside the crack following the AP de-painting process.

In terms of the effect on crack detectability, and cracks measured before and after the AP de-painting process and after LPI inspection, the measured lengths did not show different values. The cracks were clearly visible and except for a few spots of potential residue, the inside of the cracks appeared to be clear (Figure 8).

It should also be noted that the conductivity and hardness measurements were conducted before and after paint stripping treatments of the panels. The results showed negligible differences in conductivity and hardness, which illustrates that there was no considerable effect of the AP process on heat treatment and consequently the properties of the aluminium panels.

\subsection{Chemical Stripping}

A new generation of an environmentally safe stripper, EFS-2500, which has low volatility, was used in this study. The chemical paint remover was applied to the surface, left untouched for a 24-hour period of time, and then removed by scraping with soft tools and water. This paint stripping method involves minimal equipment and worker training. Figure 9(a) and Figure 9(b) demonstrate the gradual paint removal effect of the chemical process after 8 and 20 hours, respectively.

It was found that the EFS-2500 chemical solution process was successful in completely removing all paint and primer after 24 hours and left the substrate unharmed. In terms of effect on crack detectability, the close up view in Figure 10 indicates that the process left very few residues inside the crack opening. Although the cracks were not a clean as AP treated coupons, the LPI measurements were not affected. .

One may conclude that the chemical stripping is a viable process when total removal of the paint and primer is warranted, especially on relatively small areas.

\subsection{Starch Media Blasting}

The Type VII, MIL-P-85891A blasting media consisted of grains of corn starch with $5 \%$ acrylic content, which ranged in size from $100 \mu \mathrm{m}$ to $900 \mu \mathrm{m}$ as can be seen in Figure 11(a). Compressed airs at low pressures (1, 2, and 2.5 bar with a

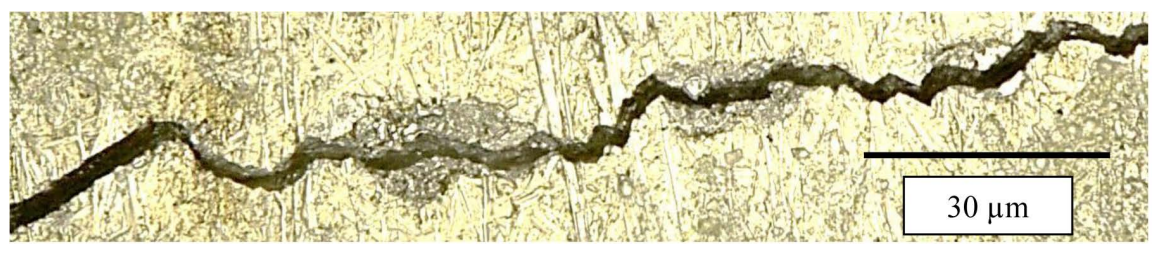

Figure 8. Close-up view of a fatigue crack in an aluminium panel section, showing almost no visible debris after plasma paint stripping. 


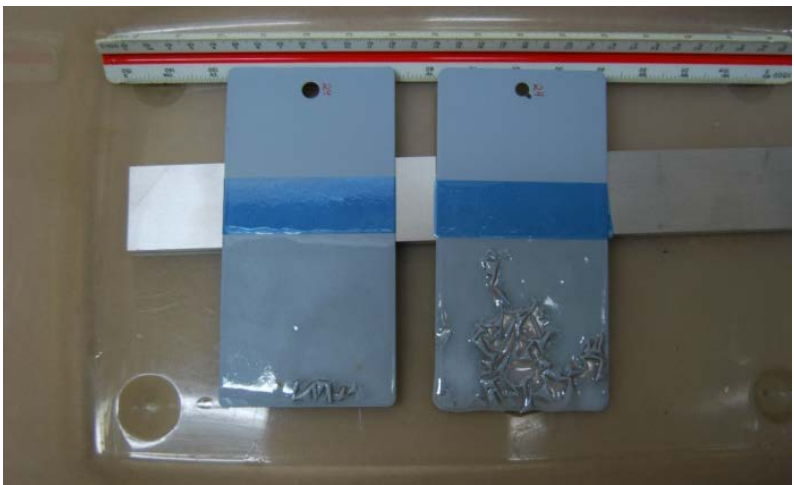

(a)

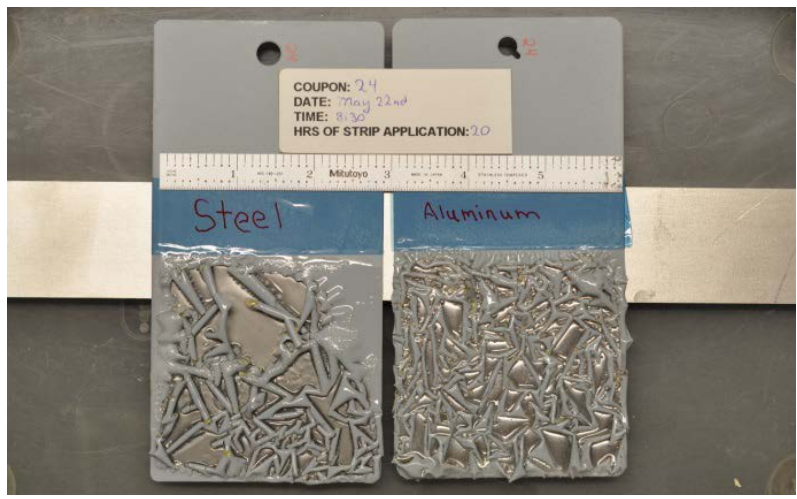

(b)

Figure 9. Top view of the coupons (Steel, Coupon 24 and Aluminium, Coupon 24) during the chemical paint removal process: (a) after 8 hours of elapsed time; (b) after 20 hours of elapsed time.

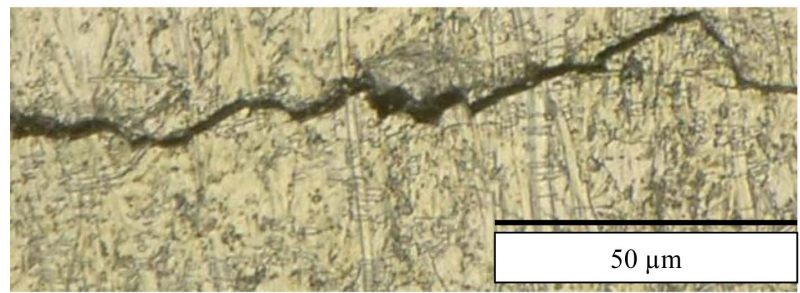

Figure 10. Close-up view of the aluminium panel sections after chemical paint removal showing the absence of residue.

mass flow rate ranging between 2.5 to $3 \mathrm{~kg} / \mathrm{min}$ ) were used to remove the coating from aluminium substrate materials suing accepted industrial practices [13].

Upon visual inspection of the stripped coupon, the paint appeared to be completely removed from the coupon, with negligible deformation to the substrate, as shown in Figure 11(b). However, Figure 11(c) displays the close-up microscopic view showing small, sporadic paint deposits found on the surface of the stripped region.

For the paint removal in the presence of cracks and its effect on crack detectability, further microscopic investigation performed on the crack paths. As can be seen in Figure 12, there were a few fine debris/residues confined inside the cracks. 


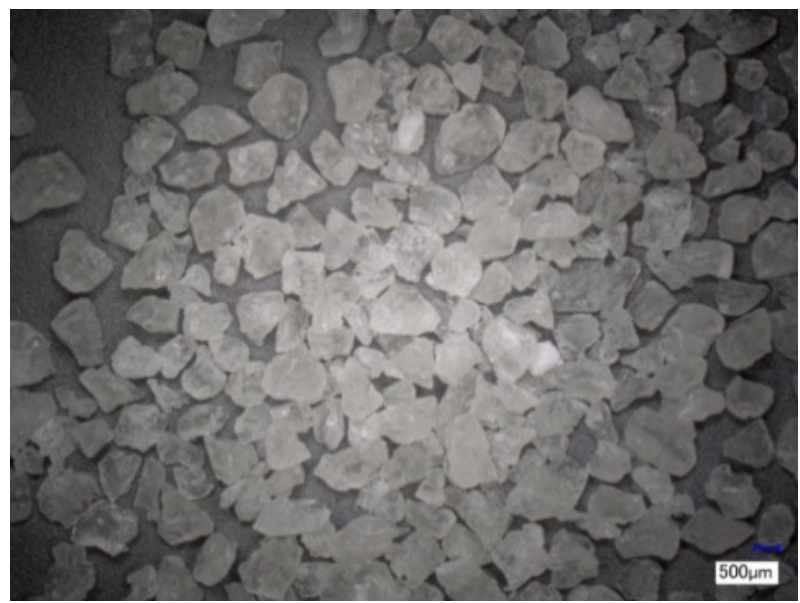

(a)

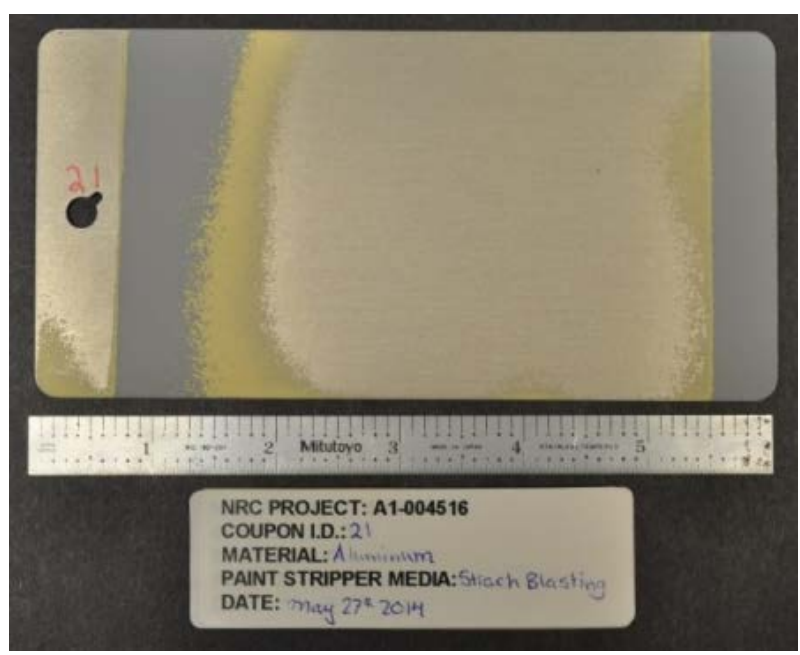

(b)

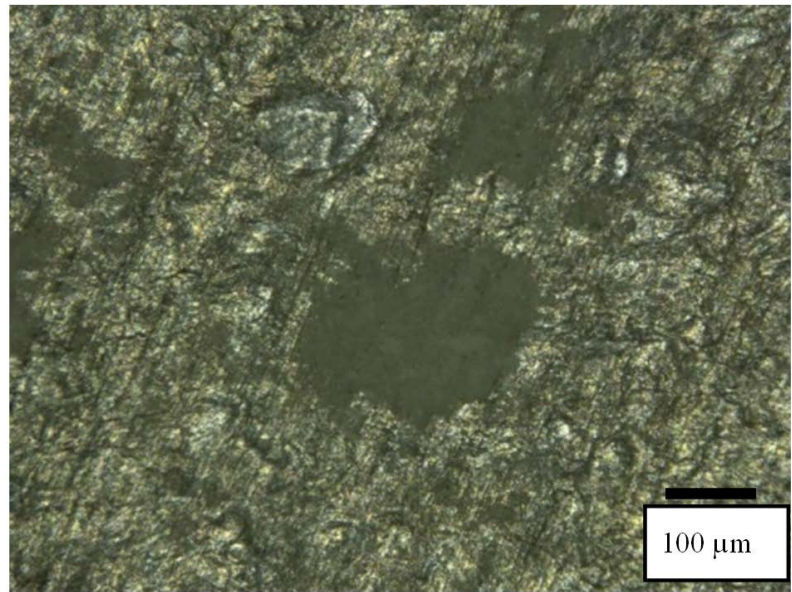

(c)

Figure 11. (a) Type VII eSritp: corn starch with 5\% acrylic content; (b) end result of starch-acrylic media blasting; (c) high magnification view of paint residues in stripped area.

Again, despite evidence of the residues entrapment inside the cracks, it appears that this method of paint removal did not negatively affect LPI in terms of 


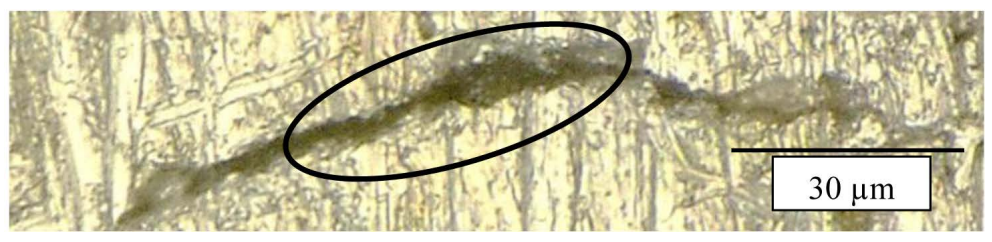

Figure 12. Close-up view of unknown residues in the cracks (circled) of the test panel 7075-T6 aluminium.

crack detection. Similar to chemical stripping, the media blasting process is also a relatively simple and efficient method for complete paint removal. The fine starch media like any blasting process, if the pressure is not controlled, could induce compressive residual stress and surface modification/warping which can shut the crack to affect LPI crack detectability.

\section{Concluding Remarks}

The effectiveness of the three paint stripping processes: chemical, Type VII acrylic starch media, and AP, was assessed using two main criteria: the extent of paint removal, and the extent of debris entrapped in the cracks, which could affect crack detectability during LPI.

First a working envelope of process parameters for the APS PlasmaFlux paint removal system was established by plasma stripping painted test panels at various plasma gun heights, speeds and number of passes per stripe. The working parameters as well as the boundary of potential damaging and ineffective parameters were identified.

From the results of this study, it is concluded that the APS PlasmaFlux system is capable of providing a multitude of combinations of parameters for removing paints. The system allows for a selective operation that can remove each layer of coating at a time. Atmospheric Plasma is a technology that has the potential to replace current hazardous and costly paint removing methods without affecting LPI crack detectability.

Post-strip macroscopic and microscopic examinations for all three processes revealed varying amounts of paint and debris residue in most of the cracks, but in general they did not appear to affect the LPI crack detectability. There was no considerable effect from any of the three processes on heat treatment and consequently, the mechanical properties of the aluminium panels.

In summary, the selection of the appropriate paint removal method for a particular aircraft structure will depend on a number of factors: purpose and the extent of paint removal, accessibility of the structure, local environment and health and safety regulations, costs, and environmental concerns. For instance, if a high level of cleanliness is required, a two-step or combination of methods may be needed to achieve the appropriate end result.

\section{Acknowledgements}

The authors would like to thank Martin Janssen and Julien Dupuis from QETE for painting all the test panels and Denis Monette from ADM Ogilvie for sup- 
porting us with Type VII starch-acrylic media blast results. We also appreciate the assistance of Mike Brothers with the EC inspections and LPI process. The present work was performed as a contribution to the DND/DRDC funded project Al-004516 “Atmospheric Plasma Paint Stripping Project”.

\section{References}

[1] Merati, A., et al. (2016) Metallographic Analysis of Paint Stripping TechniquesAtmospheric Plasma. Internal NRC Report-LTR-SMM-2016-0015. NRC, Ottawa.

[2] Merati, A., et al. (2016) Effect of Paint Removal Techniques on Crack DetectibilityLiquid Penetrant Inspection-LTR-SMM-2016-0062. NRC, Ottawa.

[3] Yancey, P. (2012) Atmospheric Plasma De-Painting. Atmospheric Plasma Solutions Inc., Cary, NC.

[4] ASTM E165/E165M-12 (2012) Standard Practice for Liquid Penetrant Examination for General Industry. ASTM International.

[5] Tracy, N. (2009) Nondestructive Evaluations (DE) Exploratory Development for Air Force Systems-AFRL-RX-WP-TR-2010-4200.

[6] Resene (2009) Primers, Sealers and Undercoats. NZIA/DBH-Resene CPD, February. http://www.resene.co.nz/archspec/cpd_earn_points/pdfs/CPD_primerssealersandu ndercoats.pdf

[7] Henkel (2016) Henkel Adhesives North America. http://na.henkel-adhesives.com/product-search-1554.htm?nodeid=8797999529985

[8] MIL-P-23377F (1989) Military Specification: Primer Coatings: Epoxy, Chemical and Solvent Resistant, S/S BY MIL-PRF-23377G.

[9] MIL-PRF-85285D (2002) Performance Specification: Coating: Polyurethane, Aircraft and Support Equipment.

[10] ASTM E1004-09 (2009) Standard Test Method for Determining Electrical Conductivity Using the Electromagnetic (Eddy-Current) Method. ASTM International.

[11] ASTM E18-16 (2016) Standard Test Methods for Rockwell Hardness of Metallic Materials. ASTM International.

[12] Yanishevsky and V. Pankov (2015) NRC Operating Procedure for APS PlasmaFlux Paint Removal System-LTR-SMPL-2015-0144. NRC, Ottawa.

[13] Foster, T. (2010) Examination of the Possible Use of Type VII-Technical Memorandum DRDC Atlantic TM 2009-293. Defence R\&D Canada-Atlantic. 
Submit or recommend next manuscript to SCIRP and we will provide best service for you:

Accepting pre-submission inquiries through Email, Facebook, LinkedIn, Twitter, etc. A wide selection of journals (inclusive of 9 subjects, more than 200 journals)

Providing 24-hour high-quality service

User-friendly online submission system

Fair and swift peer-review system

Efficient typesetting and proofreading procedure

Display of the result of downloads and visits, as well as the number of cited articles Maximum dissemination of your research work

Submit your manuscript at: http://papersubmission.scirp.org/

Or contact jmmce@scirp.org 